\title{
Model Predictive Control for Smooth Distributed Power Adaptation
}

\author{
Virgile Garcia ${ }^{1,2,3}$, Nikolai Lebedev ${ }^{1,2,3,4}$, Jean-Marie Gorce Gen $^{1,2,3}$ \\ ${ }^{1}$ University of Lyon, INRIA \\ ${ }^{2}$ INSA-Lyon, CITI, F-69621, Villeurbanne, France \\ ${ }^{3}$ INRIA-Alcatel-Lucent Bell Labs Joint Research Center \\ ${ }^{4}$ CPE Lyon, BP 2077, F-69616, Villeurbanne, France \\ Email: virgile.garcia@inrialpes.fr, lebedev@cpe.fr, jean-marie.gorce@insa-lyon.fr
}

\begin{abstract}
This paper addresses the distributed power adaptation (DPA) problem on the downlink for wireless cellular networks. As a consequence of uncoordinated local scheduling decisions in classical networks, the base stations produce mutual uncontrolled interference on their co-channel users. This interference is of a variable nature, and is hardly predictable, which leads to suboptimal scheduling and power control decisions. While some works propose to introduce cooperation between BS, in this work we propose instead to introduce a model of power variations, called trajectories in the powers space, to help each BS to predict the variations of other BS powers. The trajectories are then updated using a Model Predictive Control (MPC) to adapt transmit powers according to a trade-off between inertia (to being predictable) and adaptation to fit with capacity needs. A Kalman filter (KF) is used for the interference prediction. In addition, the channel gains are also predicted, in order to anticipate channel fading states.

This scheme can be seen as a dynamic distributed uncoordinated power control for multichannel transmission that fits the concept of self-optimised and self-organised wireless networks (SON). By using the finite horizon MPC, the transmit powers are smoothly adapted to progressively leave the current trajectory toward the optimal trajectory. We formulate the optimisation problem as the minimisation of the utility function of the difference between the target powers and MPC predicted power values. The presented simulation results show that in dynamic channel conditions, the benefit of our approach is the reduction of the interference fluctuations, and as a consequence a more accurate interference prediction, which can further lead to a more efficient distributed scheduling, as well as the reduction of the overall power consumption.
\end{abstract}

\section{Index Terms}

Distributed model-based power control, no inter-cell cooperation, power trajectory, model predictive control, smooth power adaptation, target power vs power inertia and predictability tradeoff.

\section{INTRODUCTION}

Present and future wireless networks intend to use small cells in urban environment to create local coverage to maximise the spatial reuse to support rapidly increasing data traffic. However, this dense deployment of base stations (BS) with shorter coverage range brings new challenges to the inter-cell interference management (especially at the cell boundaries) with its associated complexity. Such networks are characterised by an increasing number of distributed infrastructure elements and the lack of predictability of the base station and user patterns. Self-organized and self-optimized networks ( $\mathrm{SON}$ ) concept is therefore seen as a potential solution expected to prevent a burden of planning and optimisation tasks.

In this paper, we focus on the distributed power adaptation (DPA) problem for the downlink parallel interference channels. Radio resource power allocation has been extensively studied for many years. Recent complete overviews of the matter can be found in [1], [2], [3]. It is commonly recognised, that centralised or coordinated allocation techniques in today's classical network are more of practical issues to control the inter-BS date exchange, network latency, synchronisation aspects. The efficient power allocation in distributed systems was less studied and still presents many challenging unsolved problems. Yet, some early works on distributed radio resource management exist [4], [5], [6].

Some distributed techniques such as game theory are frameworks that, in general, require static scenarios and sequential or per-round allocation to converge to equilibrium that may not be optimum solutions. This technique 
may not be well adapted to dynamic channels. The demonstration and the conditions of the existence and uniqueness of the Generalized Nash Equilibrium are given in [7].

In most of the practical systems, the distributed radio resource management (RRM) have to rely on incomplete or altered knowledge of system or environment related parameters. For example, if a BS does not share its scheduling decisions with its neighbouring BSs, and does not have precise (i.e. accurate and up-to-date) channel state information (CSI) knowledge, then the interference received at the users is not likely to be predictable, and difficult to control.

Typically, the distributed scheduling algorithms which account for the interference in their decisions, use the parameters measured at time $n$ (channel, interference) to compute the scheduling pattern for the time $n+1$. Obviously, this would be optimal only if all other BSs kept their scheduling unchanged. Since all of them follow the same optimisation procedure independently at the same round, this leads to an intrinsically suboptimal scheduling. Some algorithms [8], [9], [10] use Kalman filter-based prediction of the interference.

Most of the approaches for resource-power allocation that can be found in the literature formulate a constrained optimisation problem to reach a target capacity or SINR, and iteratively drive the system towards the (possibly non-optimal) solution (e.g. [8], [11]). Some of them consider a trade-off between being close to this target and another metric, such as the generated interference, power level etc. [9].

To avoid the unilateral optimisation of individual BS's schedulers, one could further make an assumption on system's evolution. In this paper, we propose to apply the techniques used in automation, system assessment and trajectory tracking to follow and predict the evolution of the parameters of interest, such as the interference or power in wireless networks. Our approach is inspired by one of these techniques, which is the finite-horizon model predictive control (MPC) [12], successfully used in many industrial applications throughout the past 20 years, like process plants control in chemical industry, or production. The key idea is to look forward several steps of the optimisation, and try to get closer to a target trajectory instead of a single target state.

Instead of trying to develop a static method later extended to dynamic systems, we rather directly consider the system as dynamic. Indeed, the channel coefficients and the transmission powers evolve continuously. Thus, in our approach, we first define dynamic models that build a prior information about all the parameters in the system, such as channel gains and interference. Next, to exploit these models, we integrate in our approach three steps that have to be done by each base station: estimation, prediction and decision. In our problem investigated herein, the estimation step aims at evaluating the channel states and the interfering transmission power levels. The prediction step aims at extrapolating these values for future steps. Finally, the decision step represents the local scheduling applying a given vector of powers on the channel resources at each BS.

This last decision step is the most important contribution of this paper, as it suggests that each BS produces a partially predictable interference, performing a trade-off between its inertia and its required power variations.

This paper is organised as follows. The section II contains the model description, the section III describes the three previously mentioned processing steps. We present the simulation results in section IV, which illustrate its potential interest to gain in prediction quality and energy expenditure.

\section{Models AND NOTATIONS}

\section{A. Notations}

We use the following notations in this paper: bold upper case letters indicate matrices; bold lower case letters indicate vectors; $\mathbf{I}_{N}$ stands for identity matrix of size $N ; \mathbf{1}_{N}$ for a vector of length $N$ of ones; $\mathbf{x}^{T}$ for the transpose of $\mathrm{x}$.

\section{B. System description}

We consider a distributed cellular network with $B$ uncoordinated BSs, either because they are competitors for the channel use as it is the case for WiFi APs on ISM bands, or because the provider wishes to maximise the resource use. We consider OFDMA systems with full-reuse (Reuse 1), albeit our approach could be applied to any other system with a set of $K$ orthogonal resources. As our main concern is to provide the proof of concept, we consider the scenario where only one mobile is served by each BS, both equipped with single antenna; more elaborated scenarios are left for future work. 


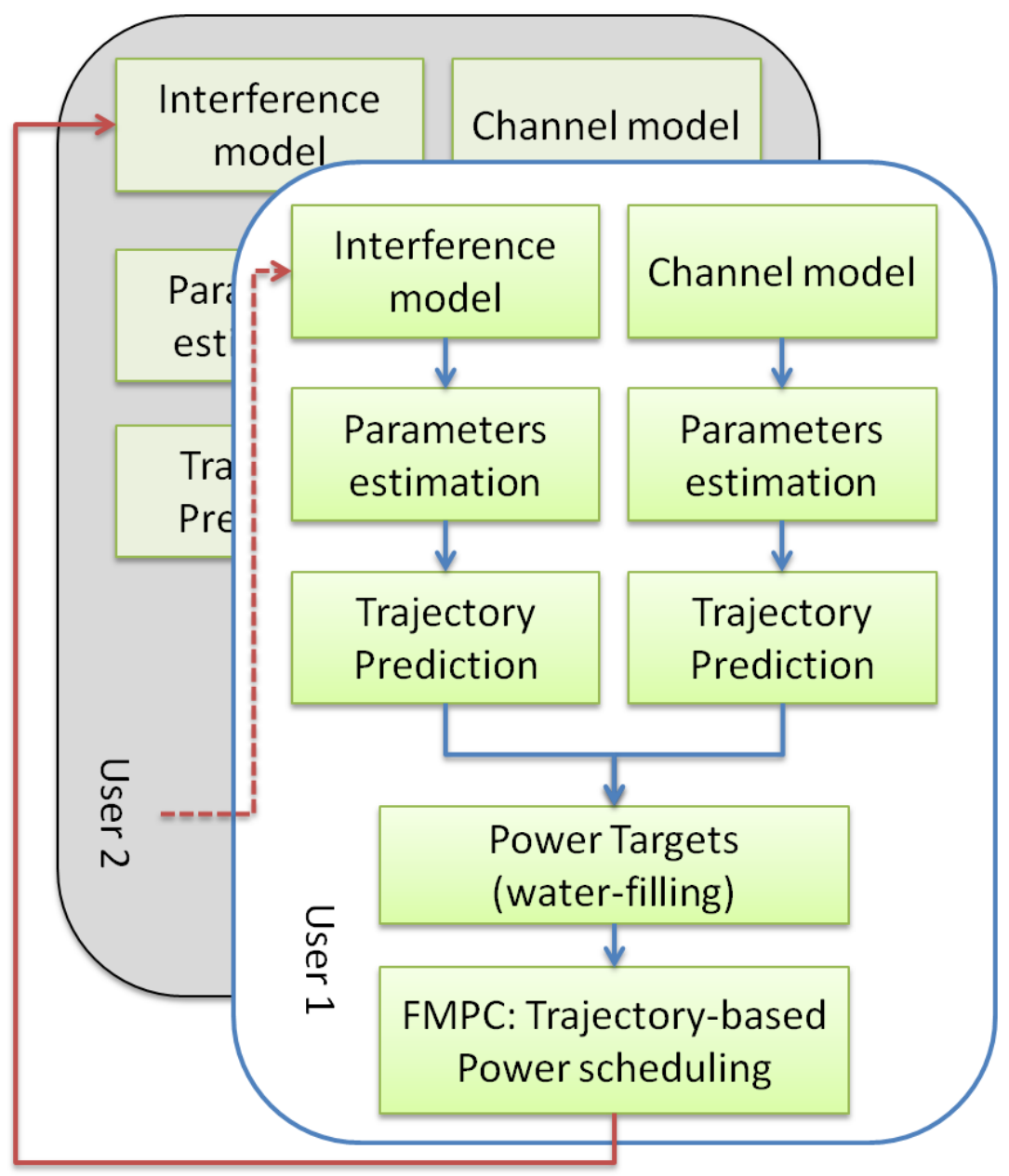

Fig. 1: Simplified system overview.

The proposed system is a framework where four fundamental elements are introduced: modelling, parameter estimation, trajectory predictions and decisions (see fig. 1). The modelling part provides the mathematical formulation for the interference, channel gains, and SINR. It is important to note that the model is a prerequisite that directly defines the three others elements of this framework. To further develop our model we assume the following capabilities:

1) Each BS broadcasts its identity and pilot signals, which are supposed orthogonal to those of other BSs.

2) Each mobile senses the interference and channel gains from the neighbouring BSs on resources and using pilot signals.

3) Each mobile feeds back its measured channel and interference levels to its serving BS only (not to all BSs).

4) Each BS estimates and predicts the future values of these parameters, and take an independent decision on the power vector for the next round. All BSs run these estimations simultaneously.

The Signal to Interference and Noise Ratio (SINR) $\gamma$ received at user $i$ on resource $k$ at time $n$ is:

$$
\gamma_{i, k}(n)=\frac{\left|h_{i, k}^{b_{i}}(n)\right|^{2} P_{k}^{b_{i}}(n)}{\sum_{b \neq b_{i}}\left|h_{i, k}^{b}(n)\right|^{2} P_{k}^{b}(n)+\sigma_{z}^{2}}
$$

where $b_{i}$ is the BS that serves the user $i$. The complex channel gain coefficient between the BS $b$ transmitting at time $n$ to user $i$ on resource $k$ with power $P_{k}^{b}(n)$ is denoted $h_{i, k}^{b}$. The received signal noise power is $\sigma_{z}^{2}$.

We aim at the model which could produce the future a priori values for some of these system parameters that can be measured. Balancing the number of parameters and the model complexity we can choose to work either on the total interference, or to separately process the channel coefficients and the transmit powers. We opt for the 
latter and jointly use two different parameter estimation models-one for the channel gains and another one for the BS transmit powers, which we now describe.

\section{Channel model: polynomial extrapolation}

We consider the time-varying fading channels, correlated in time between the consecutive channel realisations. For such a scenario, a low complexity channel model using polynomial approximation has been proposed in [13], which permits to fairly predict the next channel realisation from only few previous samples by simple extrapolation.

\section{Transmit power model: trajectory}

The originality of our approach relies on the accounting for the overall system dynamics by considering the trajectory of the power transmitted on each resource, by analogy with the spatial objects positions in target tracking or computer graphics domains. These power trajectories are considered in decibel, and at each BS we wish to predict and to track those of the other BSs, in order to smoothly adapt the BS's own transmit power, and thus to enhance the interference management within the whole system.

In this model we make an analogy between the transmit powers and the movement notations. Writing the generic $\operatorname{location}(x) / \operatorname{speed}(v) / \operatorname{acceleration}(a)$ equations in matrix form, we have at time $n$ (the time is discret with step 1):

$$
\begin{aligned}
& {\left[\begin{array}{l}
x(n+1) \\
v(n+1)
\end{array}\right]=\left[\begin{array}{ll}
1 & 1 \\
0 & 1
\end{array}\right]\left[\begin{array}{l}
x(n) \\
v(n)
\end{array}\right]+\left[\begin{array}{c}
\frac{1}{2} \\
1
\end{array}\right] a(n)} \\
& {\left[\begin{array}{l}
x(n+N) \\
v(n+N)
\end{array}\right]=\left[\begin{array}{cc}
1 & N \\
0 & 1
\end{array}\right]\left[\begin{array}{l}
x(n) \\
v(n)
\end{array}\right]+\sum_{t=1}^{N}\left[\begin{array}{c}
t-\frac{1}{2} \\
1
\end{array}\right] a(n+N-t)}
\end{aligned}
$$

In our case, the $x, v, a$ represent respectively the transmit power $P$, and its first and second derivatives over time. We deliberately choose the 2 nd order model here, so that the powers do not stay constant during the multiple future steps of prediction and the acceleration plays the role of the process noise and is therefore not predicted. This model can be generalised to higher order or even replaced by a similar one, as long as the decision steps act accordingly (see III-C).

\section{DISTRIBUTED POWER ADAPTATION}

\section{A. Parameters estimation}

In our study, the channel is computed as a polynomial regression of degree 4, based on the five last measurements (see [13] for more details). The channel measurements are supposed perfect, or otherwise any simple filter could be used prior to the extrapolation to limit the error propagation.

For the interference, we compute the trajectory of the measured values. In a more general case of several sources of interference and/or unknown channels, the trajectory of the total interference plus noise can be considered. In a more simple case with one dominant interferer, we assume that we can deduce its transmit power by dividing the estimated received interference by its respective estimated channel gain.

A Kalman filter is used to correct the measured state $\mathbf{x}$ for estimation biases or model approximations, based on the following system (here again, a general notation is used):

$$
\begin{aligned}
\mathbf{x}(n) & =\left[\begin{array}{l}
x(n) \\
v(n)
\end{array}\right] \\
\mathbf{x}(n) & =\mathbf{F} \mathbf{x}(n-1)+\mathbf{G}(n) a(n) \\
& =\mathbf{F} \mathbf{x}(n-1)+\mathbf{w}(n)
\end{aligned}
$$

with

$$
\begin{array}{r}
\mathbf{F}=\left[\begin{array}{ll}
1 & 1 \\
0 & 1
\end{array}\right], \mathbf{G}=\left[\begin{array}{c}
\frac{1}{2} \\
1
\end{array}\right] \\
\mathbb{E}\left\{a^{2}(n)\right\}=\sigma_{a}^{2} \\
\mathbb{E}\left\{\mathbf{w}(n) \mathbf{w}(n)^{T}\right\}=\left[\begin{array}{ll}
\frac{1}{4} & \frac{1}{2} \\
\frac{1}{2} & 1
\end{array}\right] \sigma_{a}^{2}
\end{array}
$$


where $\mathbf{F}$ is the state transition matrix, $\mathbf{w}$ is the process noise and $\sigma_{a}^{2}$ its variance, estimated over a moving window of previous samples, which can account for measurement error, including any inaccuracy related to hardware defaults, quantization errors, etc. In [8], the authors arbitrarily set this value to be a fraction of process noise variance, that way, the output of the Kalman filter is averaged and the system oscillations are reduced. The corrected a posteriori values are obtained using the classical Kalman filter formulation [14].

\section{B. Predictions}

The objective of the prediction part is to determine the a priori values of the channels and interference for future steps. In this work, we consider a finite-horizon based prediction, since we intend to perform scheduling not only for the next time-slot, but for a few steps forward. The ultimate objective being the smooth power adaptation, we need to track a target power over several scheduling intervals. As well, for the channels, a prediction of several future states allows us to know if a good or bad channel state will be maintained or changed quickly, and thus, make a better anticipation for future scheduling.

Based on the chosen models, once the current state parameters are defined, the future state prediction is quite straightforward. The channel gains are predicted using the aforementioned regression by computing the polynomial coefficients at time $n$ for time $n+1$ to $n+N$ ( $N$ is the horizon). Note, that a high variability of the fading process would lead to inaccurate long-term predictions: a Rayleigh channel with Doppler spread of $50 \mathrm{~Hz}$ to $100 \mathrm{~Hz}$ and $1 \mathrm{kHz}$ sampling allows about 3 future samples prediction with a reasonable quality, while lower Doppler spread $(\leq 40 \mathrm{~Hz})$ allows the prediction of 5 future states with good accuracy [13].

To predict the power trajectory, one should just put acceleration to zero in equation (2).

\section{Decisions}

Based on those predictions, the BSs will compute their transmit powers. We aim to minimise the transmit powers under user capacity constraints for parallel interference channels. This problem is a dual problem of capacity maximisation under power constraint, and when only a single user per BS is considered, a classical water-filling algorithm provides the optimal solution [1].

The resulting vector of power allocation $\mathbf{R} \in \mathbb{R}^{K N}$ is the target of the power tracking algorithm (also called "reference"). Contrarily to what is proposed in literature, we do not schedule these powers directly. Instead, we use the finite-horizon model predictive control (FMPC) [15] to smoothly track the optimal power allocation over the iterations of the global procedure. At time $n$, a cost minimising control strategy is computed for a short time horizon in the future: $[n, n+N]$. Specifically, we derive the cost-minimising trajectory that emanates from the current state until the time $n+N$. The horizon $N$ of tracking is a system parameter. In this work, we use $N=5$.

On the one hand, neighbouring users' interference prediction is based on the trajectory model, considering that $\mathbf{a}=0$. On the other hand, the local user may require a different trajectory to satisfy the optimal scheduling. The transmit powers are then subject to their inertia, and the scheduling only deviates the power vector from its initial trajectory.

On a given resource $k$, the vector containing the $N$ future transmit power values $\mathbf{P}_{k}=\left[P_{k}(n+1) \ldots P_{k}(n+N)\right]^{T}$ is given by:

$$
\mathbf{P}_{k}=\mathbf{1}_{N} P_{k}(n)+[1,2, \ldots, N]^{T} v_{k}(n)+\mathbf{W} \mathbf{a}_{k}(n),
$$

with

$$
\mathbf{W}=\left[\begin{array}{cccc}
\frac{1}{2} & 0 & \cdots & 0 \\
2-\frac{1}{2} & \frac{1}{2} & \cdots & 0 \\
\vdots & \vdots & \ddots & \vdots \\
N-\frac{1}{2} & (N-1)-\frac{1}{2} & \cdots & \frac{1}{2}
\end{array}\right]
$$

and

$$
\mathbf{a}_{k}(n)=\left[a_{k}(n), a_{k}(n+1), \ldots, a_{k}(n+N-1)\right]^{T}
$$

We perform the trade-off between the desired objective transmit powers $\mathbf{R}$ and the actually transmitted powers $\mathbf{P}$, by introducing the following utility function to minimise over $\mathbf{a}$ :

$$
U(\mathbf{a})=\sum_{k}\left(\mathbf{R}_{k}-\mathbf{P}_{k}\right)^{T}\left(\mathbf{R}_{k}-\mathbf{P}_{k}\right)+\mu_{a} \mathbf{a}_{k}^{T} \mathbf{a}_{k}
$$




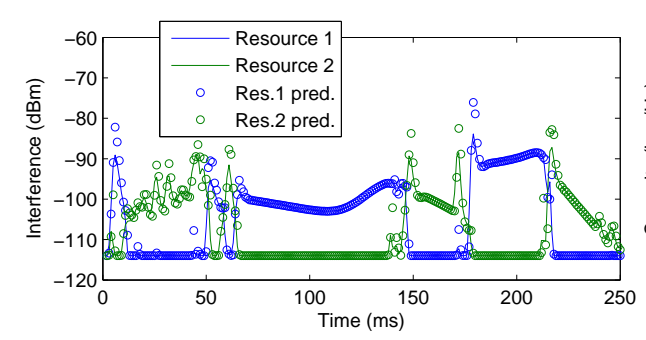

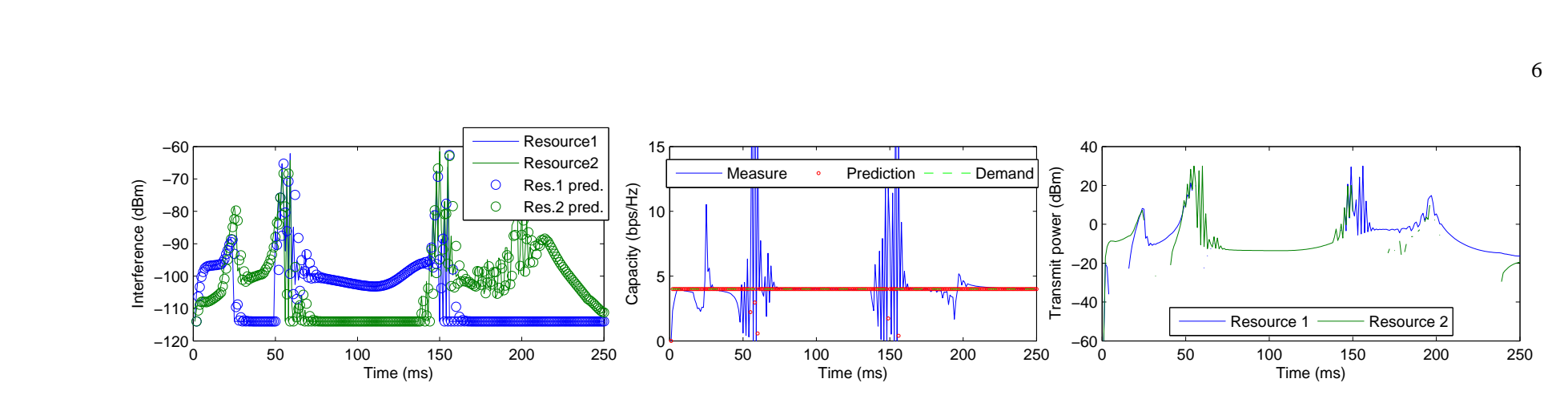

(a)
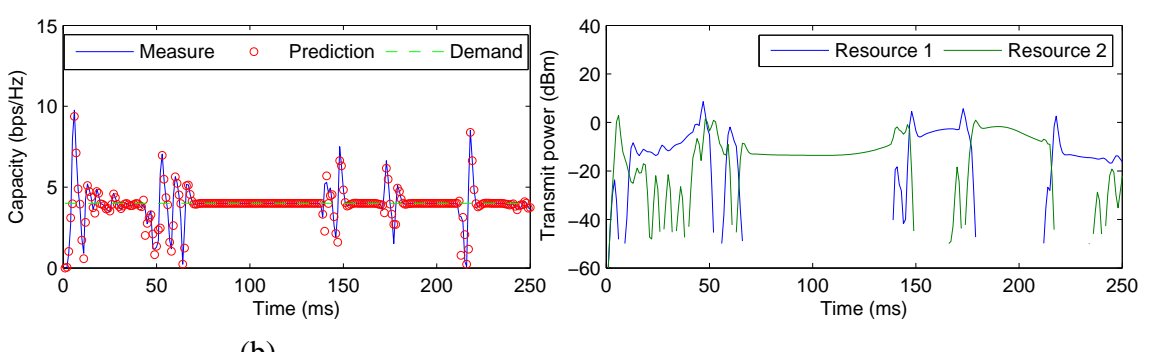

(b)
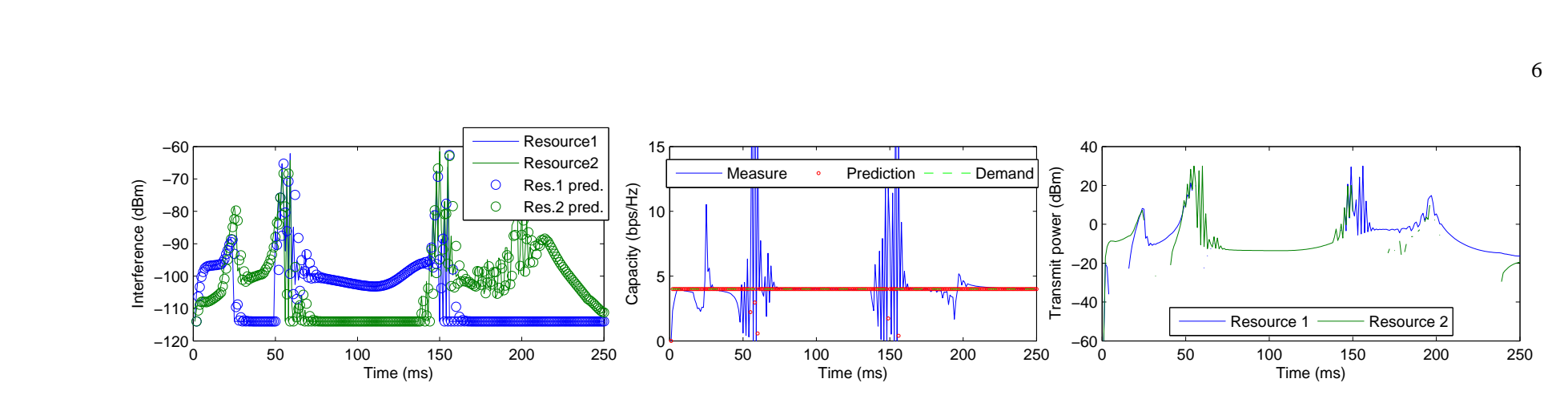

$$
\text { Hen I I H }
$$

Fig. 2: From left to right: Received interference, capacity and transmit power for a given user.

Solving the equation (12) creates a trajectory deviation that balances the optimal allocation and interference prediction. When $\mu_{a}$ is high, the importance is given on the predictability of the system, while when $\mu_{a}$ is small, a higher importance is given to try to satisfy the instant allocation. Standard systems correspond to $\mu_{a}=0$.

Lemma 1: The $\mathbf{a}_{k}$ vectors that minimise utility (12) are given, $\forall k$, by:

$$
\mathbf{a}_{k}=\left(\mathbf{W}^{T} \mathbf{W}+\mu_{a} \mathbf{I}_{N}\right)^{-1} \mathbf{W}^{T} \varepsilon_{k}
$$

where $\boldsymbol{\varepsilon}_{k}=\mathbf{R}_{k}-\mathbf{1}_{N} P_{k}(n)-[1,2, \ldots, N]^{T} v_{k}$ is the difference between the desired trajectory and the trajectory that would occur without acceleration.

Proof: The utility (12) can be reformulated as:

$$
U(\mathbf{a})=\sum_{k}\left(\varepsilon_{k}-\mathbf{W} \mathbf{a}_{k}\right)^{T}\left(\varepsilon_{k}-\mathbf{W} \mathbf{a}_{k}\right)+\mu_{a} \mathbf{a}_{k}^{T} \mathbf{a}_{k}
$$

We differentiate $U$ with respect to $\mathbf{a}_{k}$ :

$$
\frac{\partial U(\mathbf{a})}{\partial \mathbf{a}_{k}}=-2 \varepsilon_{k}^{T} \mathbf{W}+2 \mathbf{a}_{k}^{T}\left(\mathbf{W}^{T} \mathbf{W}+\mu_{a} \mathbf{I}_{N}\right)
$$

Solving for $\frac{\partial U(\mathbf{a})}{\partial \mathbf{a}_{k}}=0$ proves the Lemma 1 .

Note that even if at a given time-step the scheduler plans a trajectory with horizon, this allocation is made every time-step to accounts for environment changes.

\section{Simulation RESUlts}

For each channel, the convergence of the distributed power adaptation occurs only when the SINR demands from all users are low enough [5], even for random (unknown fading) channel [16]. In practice, considering dynamic channel conditions (mobility and fading), if the sampling is fast enough, the channel is correlated in time. Another issue is then to converge fast enough compared to the channel variation speed. Parallel channels bring here a degree of freedom for scheduling, but may increase the unpredictability of interference due to the uncoordinated decisions.

\section{A. Smooth variations}

As a first approach in this paper, we choose to study the overall system behaviour. To do so, we analyse the basic interference channel scenario: two bases stations, each serving a single user. In order to illustrate our approach, we first present a system with $K=2$ orthogonal resources available for the downlink. The mobiles are placed at half the distance between the two BSs, so that the generated interference is the major issue.

. 
We use a simulated Rayleigh channel to obtain variable gains. In this example, the target throughput for the mobiles is $4 \mathrm{bps} / \mathrm{Hz}$. Other simulation parameters are in Table I.

Figure 2 shows the comparison between the traditional systems where the distributed power allocation is updated at each time-step, using a Kalman-filter based interference estimation on fig. 2a, and our smooth power allocation method (in this example, we used $\mu_{a}=1$ ) on fig. 2b. From left to right, this figure presents, for a given mobile:

- the interference level measured and its prediction for both resources;

- the capacity measured and its prediction, compared to the actual demand;

- the transmit power used by the serving BS for both resources.

The presented result is an example over 250 iterations.

In the classical DPA, the convergence is slow and go through very unstable states. Since the interference is not predicted correctly, either the transmitter uses a power that is too low resulting in decoding errors and capacity outage, or the transmitted power is too high wasting energy and generating high interference. Note that using an estimation filter would help to reduce oscillations by averaging the measured interferences.

Comparing fig.2a and fig.2b, one can observe that the oscillations are strongly reduced in duration and amplitude. During the phases where the solutions are stable, the results are similar for both methods in terms of capacity and energy. But during transitions, or oscillations, the transmit power consumed and the capacity lost with the standard methods strongly affects overall performance (see next subsection).

The result of this is that even if the received capacity is still fluctuating around the objective (yet in a smoother way than the standard method), the prediction of the obtained capacity is much more precise: Figure 3 shows the Cumulated Density Function (CDF) of the difference between the predicted and actual capacity received at the mobiles, for various values of the parameter $\mu_{a}$. This CDF has been obtained with the values of 10000 samples for the two users considered in this scenario. Note that a too low value of $\mu_{a}$ leads to an interference model that is no longer predictable, and so applying the trajectory analogy is not relevant. A too high value of $\mu_{a}$ results in a predictable model, since powers tend to be constant, but the scheduling may no longer satisfy users requirements. Knowing the channel capacity, the transmitter can adapt the volume of data to send (if traffic allows) and thus reduce the outage.

\section{B. Power and Capacity trade-off}

We now want to show that having a more stable system leads to energy saving and capacity improvement. The scenario considered here is similar to the previous one, except that we extended the number of available resources to $K=10$, in order to have a less constrained resource allocation.

We compare the proposed model with the standard methods, i.e. Reuse- 1 and Reuse- 2 schemes, which correspond to using simultaneous water-filling updates on available resources and are similar to the Game Theory approach [7]. For Reuse-1 method and our proposed method, we apply two different estimation methods: one with a filtering estimator (which averages interference measurements), and one with non-averaged measurements (i.e. perfect measurements).

The performance metrics used are the received goodput and the transmit power used, average over the 1000 samples of the simulation. For mobile $i$, the goodput $G_{i}$ is the capacity that is successfully sent to the mobile: if the data-rate transmitted $\hat{C}_{i}(n)$ is higher than the actual capacity $C_{i}=\sum_{k} \log _{2}\left(1+\gamma_{i, k}\right)$, the data are not decodable and lost. Here, we define the goodput of the user $i$ as

$$
G_{i}(n)= \begin{cases}\hat{C}_{i}(n) & , \text { if } \hat{C}_{i}(n) \leq C_{i} \\ 0 & \text {, otherwise }\end{cases}
$$

Note that we used appropriate data-rate margin for each method, so that the goodput is optimized w.r.t. outage statistics.

\begin{tabular}{|c|c||c|c|}
\hline BS to UE distance & $200 \mathrm{~m}$ & Total noise & $-114 \mathrm{dBm}$ \\
BS to BS distance & $400 \mathrm{~m}$ & Max. Power & $30 \mathrm{dBm}$ \\
Pathloss model $(\mathrm{dB})$ & $30.18+26 \log _{10}(\mathrm{~d})$ & Min. Power & $-50 \mathrm{dBm}$ \\
Max. Doppler Shift & $10 \mathrm{~Hz}$ & Sampling freq. & $1 \mathrm{kHz}$ \\
\hline
\end{tabular}

TABLE I: Simulation Parameters 


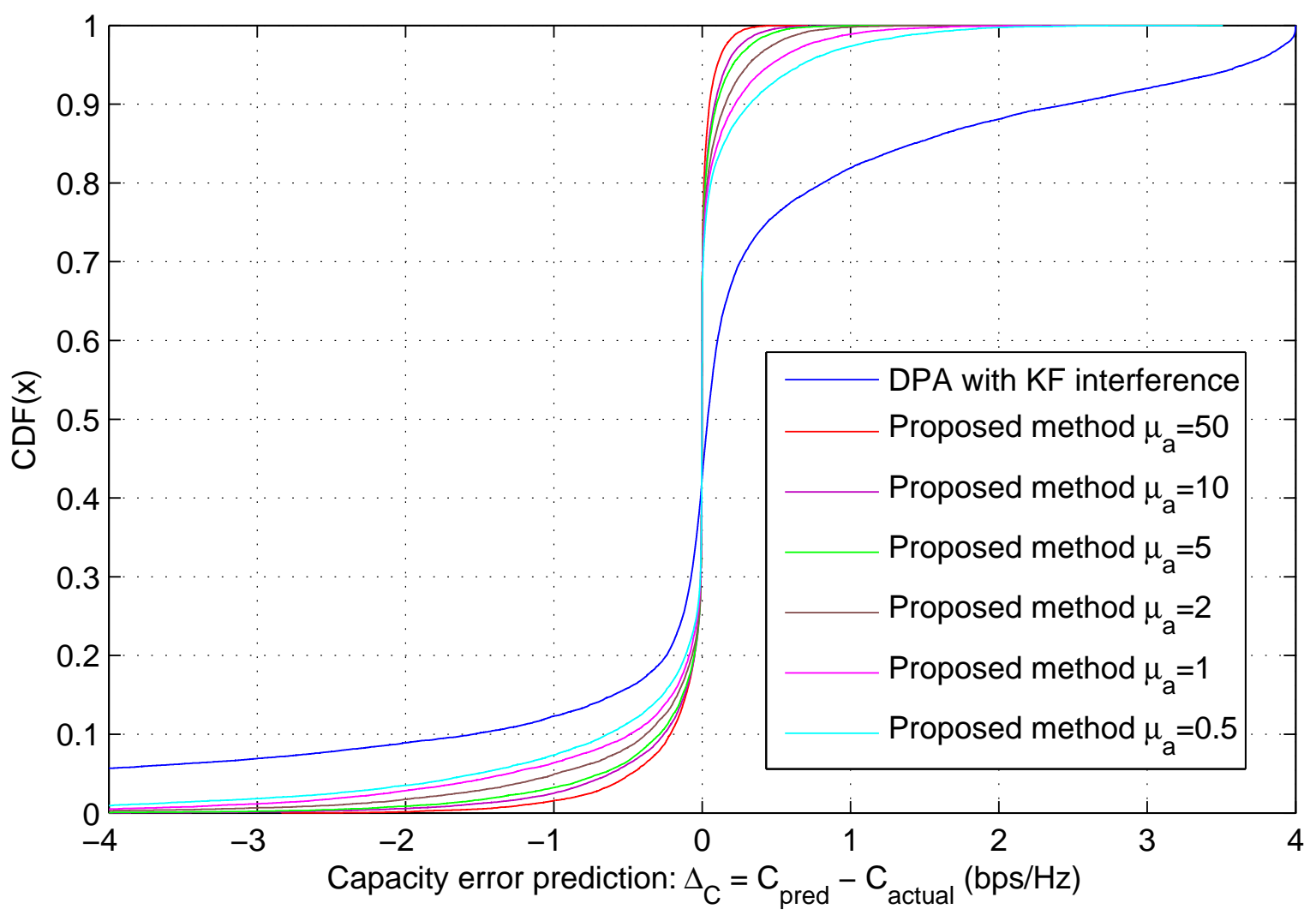

Fig. 3: CDF of the capacity prediction error.

Figure 4 shows the performance of the compared methods, with various capacity requirements. It can be seen that the proposed method outperforms the Reuse- 2 by about $2 \mathrm{~dB}$ energy gain for low capacity requirements. Our method also outperforms the standard method as soon as the interference is high enough to create convergence issues. This gain in energy comes from the usage of the appropriate level of power for transmissions, due to improved predictions and right usage of frequency diversity.

For higher capacity requirements, the classical method is bounded by a maximum achievable rate after which any further requirement leads to higher interference level and oscillations, and so can not improve the system. Using filtered measurements, these oscillations are reduced and so the overall performance is improved, yet still affected by frequent outage and energy waste, due to wrong predictions. Subject to fewer wrong predictions, the proposed method allows to improve both goodput and energy consumption and obtains results closer to the Reuse- 2 reference. Note that Reuse- 2 is an extreme case since it is not affected by any interference and so has no convergence issue.

It is shown that averaging the interference measurement of our method brings no significant improvement, which confirms that the trajectory-based approach scheduling intrinsically acts as a smooth power allocation.

\section{CONCLUSION}

In this paper, we presented a framework for dynamic distributed power allocation in parallel interference channels, which does not require information sharing among transmitters. We adapted the finite-horizon model predictive control to the power tracking, in order to derive the distributed scheduling so that the generated interference is kept tightly within a predefined model. We have shown that, using a trajectory modelling of the channel and interference as an a priori knowledge on system behaviour, improves the interference management and capacity prediction, and thus leads to finer power allocations.

In a future work, we aim to look at theoretical stability regions, as well as consider multiple users and multiple cells to extend this model to more realistic scenarios. 


\section{ACKNOWLEDGEMENT}

This work has been carried out in the frame of the joint lab between INRIA and Alcatel-Lucent Bell Labs on "Self Organizing Networks".

\section{REFERENCES}

[1] Mung Chiang, Prashanth Hande, Tian Lan, and Chee Wei Tan. Power control in wireless cellular networks. Found. Trends Netw., 2:381-533, April 2008.

[2] Vaggelis G. Douros and George C. Polyzos. Review of some fundamental approaches for power control in wireless networks. Computer Communications, 34(13):1580 - 1592, 2011.

[3] S. Koskie and Z. Gajic. "signal-to-interference-based power control for wireless networks: A survey, 1992-2005". Dynamics of Continuous, Discrete and Impulsive Systems B: Applications and Algorithms, 13, no. 2:187-220, 2006.

[4] J. Zander. Distributed cochannel interference control in cellular radio systems. Vehicular Technology, IEEE Transactions on, 41(3):305 -311 , aug 1992.

[5] G.J. Foschini and Z. Miljanic. A simple distributed autonomous power control algorithm and its convergence. Vehicular Technology, IEEE Transactions on, 42(4):641 -646, nov 1993.

[6] J.C.-I. Chuang and N.R. Sollenberger. Performance of autonomous dynamic channel assignment and power control for tdma/fdma wireless access. Selected Areas in Communications, IEEE Journal on, 12(8):1314 -1323, oct 1994.

[7] Jong-Shi Pang, G. Scutari, F. Facchinei, and Chaoxiong Wang. Distributed power allocation with rate constraints in gaussian parallel interference channels. Information Theory, IEEE Transactions on, 54(8):3471 -3489, aug. 2008.

[8] K. K. Leung. Power control by interference prediction for broadband wireless packet networks. 1(2):256-265, 2002.

[9] S. Sorooshyari and Z. Gajic. Autonomous dynamic power control for wireless networks: User-centric and network-centric consideration. Wireless Communications, IEEE Transactions on, 7(3):1004 -1015, march 2008.

[10] K. Shoarinejad, J. L. Speyer, and G. J. Pottie. Integrated predictive power control and dynamic channel assignment in mobile radio systems. IEEE Journal on Wireless Communications, 2(5):976-988, 2003.

[11] Hui Zhang, Jingya Li, Xiaodong Xu, T. Svensson, C. Botella, and Sangyun Lee. Channel allocation based on kalman filter prediction for downlink ofdma systems. In Vehicular Technology Conference Fall (VTC 2009-Fall), 2009 IEEE 70th, pages 1 -4, sept. 2009.

[12] Michael Nikolaou. Model predictive controllers: A critical synthesis of theory and industrial needs. Advances in Chemical Engineering, 26:131-204, 2001.

[13] Zukang Shen, J.G. Andrews, and B.L. Evans. Short range wireless channel prediction using local information. In Signals, Systems and Computers, 2003. Conference Record of the Thirty-Seventh Asilomar Conference on, volume 1, pages 1147 - 1151 Vol.1, nov. 2003.

[14] Greg Welch and Gary Bishop. An introduction to the kalman filter. In Proc of SIGGRAPH, Course 8, 2001.

[15] E. Camponogara, D. Jia, B. H. Krogh, and S. Talukdar. Distributed model predictive control. IEEE Control Systems, 22(1):44-52, 2002.

[16] Tim Holliday, Nick Bambos, Peter Glynn, and Andrea Goldsmith. Distributed power control for time varying wireless networks: Optimality and convergence. In in Proceedings: Allerton Conference on Communications, Control, and Computing, 2003. 


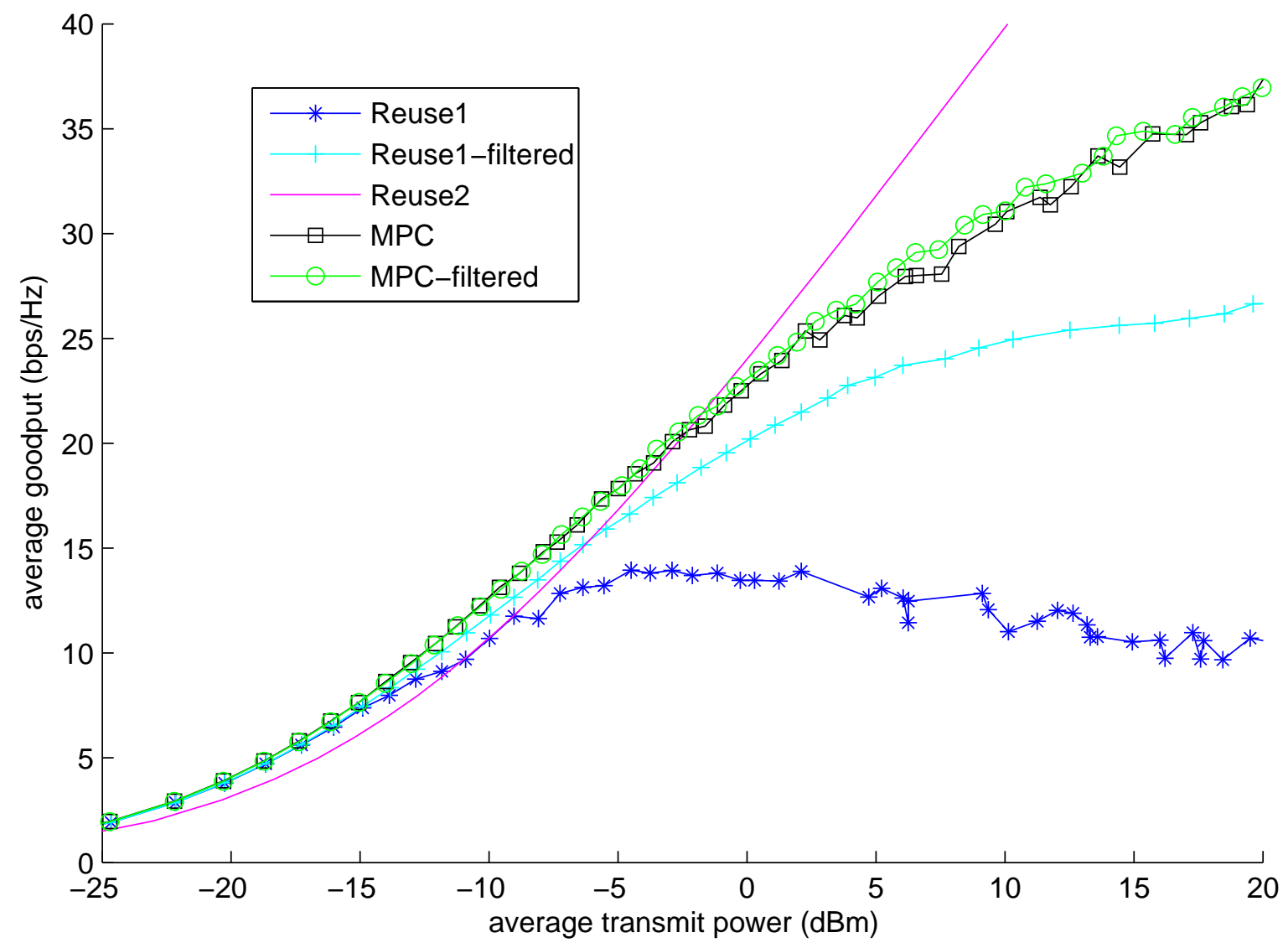

Fig. 4: Capacity versus power consumption results. 